\title{
A new amorphous ribbon fluxgate sensor based on torsional-creep-induced anisotropy
}

Nielsen, Otto V.; Gutierrez, J.; Hernando, B.; Savage, Howard T.

Published in:

I E E E Transactions on Magnetics

Link to article, DOI:

$10.1109 / 20.50554$

Publication date:

1990

Document Version

Publisher's PDF, also known as Version of record

Link back to DTU Orbit

Citation (APA):

Nielsen, O. V., Gutierrez, J., Hernando, B., \& Savage, H. T. (1990). A new amorphous ribbon fluxgate sensor based on torsional-creep-induced anisotropy. I E E E Transactions on Magnetics, 26(1), 276-280.

https://doi.org/10.1109/20.50554

\section{General rights}

Copyright and moral rights for the publications made accessible in the public portal are retained by the authors and/or other copyright owners and it is a condition of accessing publications that users recognise and abide by the legal requirements associated with these rights.

- Users may download and print one copy of any publication from the public portal for the purpose of private study or research.

- You may not further distribute the material or use it for any profit-making activity or commercial gain

- You may freely distribute the URL identifying the publication in the public portal

If you believe that this document breaches copyright please contact us providing details, and we will remove access to the work immediately and investigate your claim. 


\title{
A New Amorphous Ribbon Fluxgate Sensor Based on Torsional-Creep-Induced Anisotropy
}

\author{
OTTO V. NIELSEN, J. GUTIERREZ, B. HERNANDO, AND HOWARD T. SAVAGE
}

\begin{abstract}
The fluxgate sensor presented here consists of a hairpin shaped nonmagnetostrictive amorphous metal ribbon, which carries the excitation ac current. Even harmonics are induced in a surrounding coil which may be used both as a pick-up coil and as a field compensation coil. The principle of operation is similar to that of a traditional ring-core system except that the core itself carries the excitation current. The present sensor competes favorably with traditional fluxgate sensors with respect to simplicity, miniaturization, signal level, and noise level.
\end{abstract}

\section{INTRODUCTION}

$I^{2}$ N FLUXGATE magnetometers the sensor element often consists of a magnetic ring core (see Fig. 1) which is magnetically excited by an ac current flowing in a surrounding toroidal coil. This arrangement is placed in a detector coil which also may function as a feedback coil for compensation of the external magnetic field to be measured. The core consists of a nonlinear ferromagnetic material which generates a fundamental as well as harmonics in the ring-core flux.

In the absence of an external field (signal field), the signal induced in the detector coil is zero by symmetry. If a finite field is present with a nonzero component along the detector coil axis, even harmonics will be induced in the detector coil. Most fluxgate systems utilize the second harmonic as the signal voltage analyzed by the accompanying electronics.

Other types of fluxgate sensors have been described in the literature. For a more exhaustive list see Gordon $e t$ al. [1] and Primdahl [2]. For the present purpose we draw attention to a few sensor configurations. A widely used sensor is the Vacquier sensor which has two rod-shaped cores surrounded by one detector coil [3]. Another two-

Manuscript received May 12, 1989; revised August 11, 1989. The work of J. Gutierrez was supported under a Grant from the Basque Government. The work of B. Hernando was supported by the Direccion General de In vestigacion Cientifica y Technica.

O. V. Nielsen is with the Department of Electrophysics, The Technical University of Denmark, DK-2800 Lyngby, Denmark.

J. Gutierrez is with the Department of Electrophysics, The Technical University of Denmark, DK-2800 Lyngby, Denmark, on leave from Facultad de Ciencias, Universidad del Pais Vasco, 48080 Bilbao, Spain.

B. Hernando is with the Department of Electrophysics, The Technical University of Denmark, DK-2800 Lyngby, Denmark, on leave from the Departamento de Fisica, Universidad de Oviedo, Oviedo, Spain.

H. T. Savage was with the Department of Electrophysics, The Technical University of Denmark, DK-2800 Lyngby, Denmark. He is now with the U.S. Naval Surface Weapons Center, White Oak, Silver Spring, MD 20910.

IEEE Log Number 8931790.

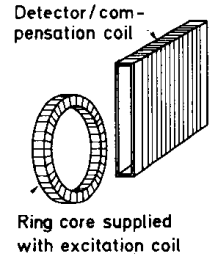

Fig. 1. Sensor element for a ring-core fluxgate magnetometer.

core sensor is the Foerster sensor which has two serial coupled detector coils each surrounding one core [4]. These sensors have in common with the ring-core sensor "parallel-gating"' elements in the sense that the signal field is parallel to the excitation field.

Various "orthogonally gated" systems have also been proposed. Palmer [5] described one configuration of this type. Here the magnetic core consists of a ferromagnetic wire carrying an excitation current that produces a circular field perpendicular to the wire axis. The detector coil is aligned coaxially with the magnetic wire. In another configuration described by Alldredge [6] the topology is similar to that in Fig. 1 except that the axis of the detector coil is colinear with the axis of the torus.

Schonstedt [7] introduced a mixed orthogonal/parallel type which may be described in the following way. Consider a (soft) ferromagnetic wire material which is wound as a helix. Let the helix be exposed only to a (small) circular magnetic field generated by an ac current flowing through a straight wire situated along the centerline of the helix. Due to the shape anisotropy of the ferromagnetic wire this will be ac magnetized almost parallel to its wire axis thereby giving rise to a magnetization component parallel to the helix axis. A detector coil surrounding the helix can thus pick up a signal induced by this parallel component. This signal contains only odd harmonics of the fundamental frequency. The sensor described by Schonstedt contains two helixes oppositely wound on the same support. This configuration gives rise to a cancellation of the odd harmonics induced individually by the two helixes. The effect of an external field component parallel to the helix axis is to break the symmetry so that noncanceling even harmonics occur.

The fluxgate sensor described in the present paper resembles to some extent the sensor introduced by Schonstedt. But instead of making use of the shape anisotropy to produce a magnetization component parallel to the ex-

0018-9464/90/0100-0276\$01.00 (C) 1990 IEEE 
citation current, we use an intrinsic anisotropy for the same purpose. Furthermore, we use the ferromagnetic core material itself as the carrier of the excitation current.

\section{PRINCIPLE OF OPERATION}

The ferromagnetic core consists of a nonmagnetostrictive metallic glass ribbon which has been heat-treated under mechanical torsion so that a "helical" magnetic anisotropy, i.e., an anisotropy with axes tilted with respect to the ribbon axis, has been induced. A material with zero magnetostriction has been chosen in order to ensure a high permeability. After the heat treatment the ribbon is given a hairpin shape, see Fig. 2. In the figure, the directions of easy axes of magnetization are indicated by lines inclined $45^{\circ}$ to the ribbon axis. The lines shown correspond to the easy axes for that half of the material which is below the visible surface. In the other half, the easy axes are also inclined $45^{\circ}$ to the ribbon axis, but they are perpendicular to the first ones as shown enlarged in the figure.

Assume a current $I$ flowing through the ribbon in the direction indicated by the arrows. Inside the ribbon this current will set up a circular magnetic field which forces the magnetization to form ideally one magnetic domain in one half of the ribbon and another magnetic domain in the other half of the ribbon. Initially the magnetization vectors of these domains will be inclined $45^{\circ}$ to the ribbon axis, thus giving rise to a net magnetization along the ribbon axis in the direction of the current. By increasing the current, the domain magnetizations will gradually point more and more perpendicular to the ribbon axis thus decreasing the net ribbon axis magnetization.

In our sensor, the coil shown in Fig. 2 surrounds the hairpin-shaped ribbon, and in the magnetization process described above the net flux through the coil will be zero. Now, assume the current to be alternating while an external dc field is present with a nonzero ribbon axis component. In this case the alternating ribbon axis magnetization will induce even harmonics in the coil. In our fluxgate measurements we make use of the second harmonic produced in this way.

\section{Construction Data}

A number of sensors were prepared as described below. As core material was used VITROVAC 6025 from Vacuumschmelze $\mathrm{GmbH}$, Hanau, in ribbon form, $1.0 \mathrm{~mm}$ $\times 0.0226 \mathrm{~mm}$ in cross section. This Co-rich material is characterized by a very low magnetostriction coefficient which was measured to $-0.18 \times 10^{-6}$ for the as-received ribbon.

This (negative) value is in rough agreement with that $\left(-0.12 \times 10^{-6}\right)$ measured by O'Dell [8]. Other characteristics for the material is a very low coercivity $(<2.4$ $\mathrm{A} / \mathrm{m}$ ) and a saturation polarization $J_{s}=0.55 \mathrm{~T}$. Further material properties of interest are the high electrical resistivity of $135 \mu \Omega \cdot \mathrm{cm}$ and the Youngs modulus of 150 $\mathrm{GPa}$.

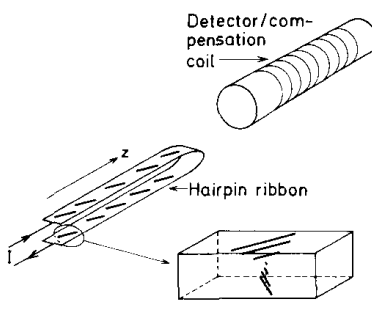

Fig. 2. Sensor element for the present fluxgate magnetometer. The enlarged part of the hairpin ribbon illustrates the direction and the magnitude of the induced anisotropy.

Recent investigations [9] on other Co-rich nearly nonmagnetostrictive materials have shown that annealing in the temperature range $300^{\circ} \mathrm{C}-400^{\circ} \mathrm{C}$ has the effect of increasing the magnetostriction about $0.25 \times 10^{-6}$ toward more positive values. The same turns out to be the case for the present VITROVAC ribbons. The presence of a small negative magnetostriction in the as-quenched VITROVAC is thus an advantage for the present use of the ribbon because we need heat treatments in this temperature range for other reasons. In fact, our heat treatments on the VITROVAC results in a very small positive magnetostriction coefficient $\left(0.06 \times 10^{-6}\right)$.

In order to induce the helical anisotropy mentioned above, the ribbons were annealed under conditions chosen on the basis of previous experiments on other zero or nearly zero magnetostriction alloys of similar compositions [10], [11]. These experiments have shown that a magnetic anisotropy in an amorphous ribbon can be induced by exposing the ribbon to a tensile-stress annealing. An important result to be used here is that the anisotropy is not related directly to the macroscopic magnetostriction, i.e., the anisotropy can be induced in magnetostrictive as well as in nonmagnetostrictive alloys. Another result is that the induced anisotropy is proportional to the tensile stress applied during the annealing treatment, and for the materials in question it is of the order of $0.7 \mathrm{~J} / \mathrm{m}^{3}$ per megapascal stress applied for $1 \mathrm{~h}$ at temperatures between $300^{\circ} \mathrm{C}$ and $400^{\circ} \mathrm{C}$. At these conditions the tensile axis will be the hard magnetic axis.

In the annealing of our sensor ribbons we twisted the ribbons $74 \mathrm{rad} / \mathrm{m}$ giving rise to a shear stress of $100 \mathrm{MPa}$ near the surface. This shear stress is equivalent to mutual perpendicular tensile and compressive stresses of $100 \mathrm{MPa}$ acting along the (principal) axes tilted $45^{\circ}$ with the ribbon axis. Furthermore, we assume that the effect on the induced anisotropy of a compressive stress is similar (apart from the sign) as that of the tensile stress. We therefore expect the effective stress to be a tensile stress of $200 \mathrm{MPa}$ near the surface decreasing linearly to zero in the center plane. This is the initial stress pattern, but during the annealing process $\left(1 \mathrm{~h}\right.$ at $\left.340^{\circ} \mathrm{C}\right)$ the ribbon attains a viscoplastic deformation (creep) which has the effect of decreasing the applied stress. From the final ribbon shape we estimate this effect to reduce on average the effective applied stress by a factor of 3 to about $67 \mathrm{MPa}$. 
In order to keep the ribbon "straight" when twisted it was necessary to apply a tensile stress of $32 \mathrm{MPa}$ along the ribbon axis. This has the effect of turning the easy axes of magnetization above $45^{\circ}$ from the ribbon axis.

Apart from the latter effect, our prepared sensor ribbons have intrinsic anisotropies with easy axes inclined $45^{\circ}$ to the ribbon axis. The axes on either side of the center plane are mutually perpendicular. The magnitude of the anisotropy constant $K$ below the surface is estimated to $67 \times$ $0.7=47 \mathrm{~J} / \mathrm{m}^{3}$ which is equivalent to an anisotropy field $H_{K}=2 \mathrm{~K} / J_{s}=171 \mathrm{~A} / \mathrm{m}=2.1 \mathrm{Oe}$.

Fig. 3 shows, for a prepared sensor ribbon, the ribbon axis polarization $J_{z}$ as a function of the ribbon current density $I$ measured at $20 \mathrm{~Hz}$. From the simple anisotropy model shown in Fig. 2 we expect a remanence of $(\sqrt{2} / 2) J_{s}$. The actual remanence, however, only amounts to about 60 percent of this value. We explain this reduction by the above mentioned increase of anisotropy angle due to the tensile stress applied during the annealing process.

Assuming for simplicity the $45^{\circ}$ model we may furthermore calculate from Fig. 3 the magnitude of the induced anisotropy. It is easily shown that $K$ below the surface is given by

$$
K=(\sqrt{2} / 4) a J_{s}^{2}\left(d J_{z} / d I\right)^{-1}
$$

where $a$ is the half thickness of the ribbon and $d J_{z} / d I$ is the slope of the magnetization curve near $I=0$. From Fig. 3 we find in this way $K=40 \mathrm{~J} / \mathrm{m}^{3}$ in reasonable agreement with our estimates.

For practical reasons the ribbon is cut in two halves which are soldered on each side of a double-sided print board etched with suitable terminals and cut as a suitable long shape. The free length of each half-ribbon is $60 \mathrm{~mm}$. The measurements referred to below are made with a 46$\mathrm{mm}$-long detector coil wound with $0.1-\mathrm{mm} \mathrm{Cu}$ wire on a $3,0-\mathrm{mm}$ suction tube surrounding the ribbon. The number of turns is 424 .

\section{Sensor Performance}

Investigations were performed with the sensors situated in a six-layer mu-metal shielding box. A small magnetic field could be applied by a coil situated inside the shield. Using a lock-in amplifier we studied the second-harmonic sensor output for various applied fields and exciting ac currents. The fundamental frequency was varied from 0.5 to $20 \mathrm{kHz}$.

In order to avoid excessive heating, the exciting current density was not allowed to exceed $8.8 \mathrm{~A} / \mathrm{mm}^{2}$, which was necessary to apply at the highest frequencies in order to get reproducible results. The need for a high current at the high frequencies stems from a broadening of the magnetization curve (Fig. 3) due to anomalous eddy currents. Because of this broadening, the high current is necessary in order to obtain saturation in each cycle.

Fig. 4 shows the second-harmonic output at $15 \mathrm{kHz}$ as a function of the applied field for one of the sensors. Ob-

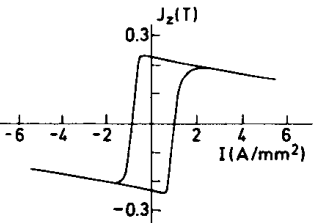

Fig. 3. Ribbon axis magnetization as a function of the current flowing through the ribbon. Notice that the net magnetization is parallel to the current.

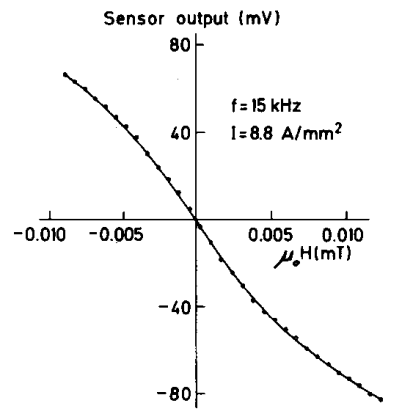

Fig. 4. Open-circuit sensor output as a function of applied field parallel to the ribbon axis. Detector coil: 424 windings.

viously the output is strongly nonlinear with respect to the field above $0.004 \mathrm{mT}$. This is a general trend for all frequencies and exciting currents, which means that the sensor is usable only as a zero-detecting element in a device like a fluxgate magnetometer. Defining the sensitivity of the sensor as the slope of the sensor output versus field curve when passing zero, we find from Fig. 4 a sensitivity of $10 \mathrm{~V} / \mathrm{mT}$.

The frequency dependence of the sensitivity for a fixed excitation current of $8.8 \mathrm{~A} / \mathrm{mm}^{2}$ is shown for one sensor in Fig. 5. The tendency of saturation for the sensitivity at higher frequencies is expected from eddy-current effects. At low frequencies $(<10 \mathrm{kHz})$, however, we expected the sensitivity to be proportional to the frequency. This is in sharp contrast to the low-frequency behavior revealed in Fig. 5.

The noise level was studied for all the sensors. Fig. 6 shows for the best sensor the response measured with 0.5$\mathrm{Hz}$ bandwidth, to a square-wave field of peak-peak amplitude of $6 \mathrm{nT}$. Performing a Fourier analysis of this sensor output we find a noise level of $0.21 \mathrm{nT} / \sqrt{\mathrm{Hz}}$ in the frequency range $0.1-0.5 \mathrm{~Hz}$. The noise spectrum shows typical $1 / f$ behavior with a decrease of 1.3 decades per decade. All the sensors revealed this type of noise behavior with levels below $1 \mathrm{nT}$, typically $0.4 \mathrm{nT}$ in the range $0.1-1.0 \mathrm{~Hz}$.

Usually the output signal has a finite value even for zero applied field. This is due to inevitable differences of geometry and physical properties of the two halves of the hairpin core. Defining the offset as the field necessary to cancel this signal, we find typically offset values of 30 $\mathrm{nT}$. However, the offset is easily adjusted to zero by ad- 


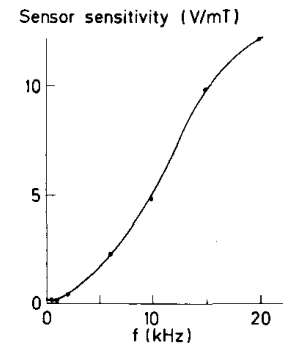

Fig. 5. Frequency dependence of the sensor sensitivity defined as $d$ (output) $/ d$ (field) for field $=0$.

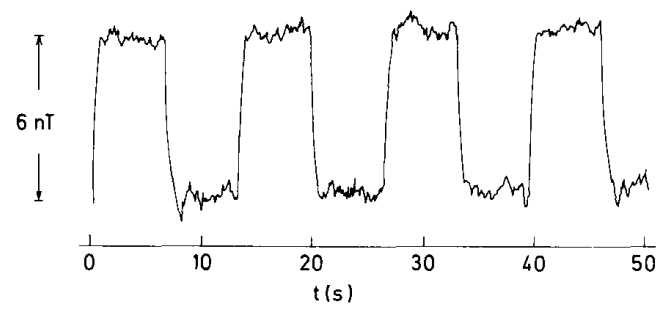

Fig. 6. Signal output at $0.5-\mathrm{Hz}$ bandwidth. The sensor is exposed to a 6-nT square-wave applied field.

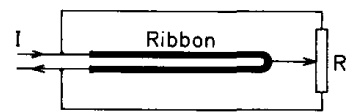

Fig. 7. Offset adjustment by means of a potentiometer.

justing the exciting current through a potentiometer as shown in Fig. 7. This method of offset adjustment was used in most of the experiments described above.

\section{Theoretical Considerations}

In order to elucidate the mode of operation, we use a simple theory for this magnetometer. We assume a demagnetized structure such as that in Fig. 8. For purposes of this theory this domain configuration is consistent with the domain observations of Livingston and Morris [12] in twisted ribbons. The domain marked " 1 " is to be typical of the domains in volume $v_{1}$ of the sample with respect to the applied fields. The domain marked " 2 "' is likewise typical of the domains in volume $v_{2}$.

Now, assume a small applied field along the ribbon axis. To a first approximation, we consider the magnetization angle $\theta$ constant, i.e., not dependent on the distance $x$ from the center plane of the ribbon. The $z$ component of the polarization is then

$$
J_{z}=J_{s}\left\langle\left(v_{1}-v_{2}\right) /\left(v_{1}+v_{2}\right)\right\rangle \cos \theta=J_{s} \Delta \cos \theta
$$

where $J_{s}$ is the saturation polarization of the ribbon.

At a distance $x$, the anisotropy constant due to the twist annealing and subsequent flattening of the ribbon is assumed to be $K(x / a)$ thereby giving rise to an energy den-
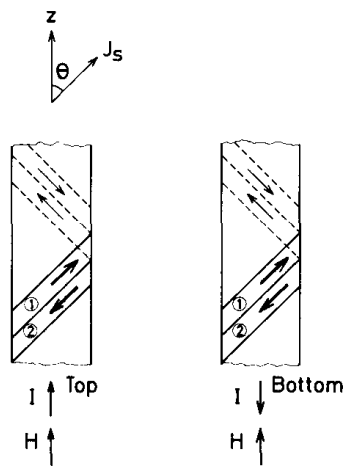

Fig. 8. Domain configuration used in the text.

sity

$$
E_{K}=-K(x / a) \cos ^{2}(\theta-\pi / 4) .
$$

The demagnetizing energy density is

$$
E_{D}=(1 / 2) D \mu_{0}^{-1} J_{s}^{2} \Delta^{2} \cos ^{2} \theta
$$

where $D$ is the demagnetization factor. The Zeeman energy is

$$
E_{H}=-H J_{s} \Delta \cos \theta
$$

due to the unknown field $H$ and

$$
E_{l}=-I x J_{s} \Delta \sin \theta
$$

due to the current density $I$. The total energy density $E_{\text {tot }}$ is thus

$$
E_{\text {tot }}=E_{K}+E_{D}+E_{H}+E_{I} .
$$

In order to avoid hysteresis effects the sensor must be operated under saturated conditions, i.e., $J_{z}$ as given by (1) must oscillate in the full range $-J_{s} \cos \theta$ to $+J_{s} \cos \theta$ with $\theta$ close to $\pi / 4$. The driving force is represented by the Zeeman energy [5] from the ribbon current. The energy mainly responsible for this $z$-axis oscillation is the helical anisotropy energy given by (2). This shows the importance of helical anisotropy in a sensor of this construction.

For a prediction of magnetometer sensitivity we follow Primdahl et al. [13] and calculate the short-circuit, output pulses. We assume a rapid change in current density $I$, consequently giving rise to a rapid change $\delta J_{z}$ in the sum of the polarizations of the two parts of the ribbon carrying the current in opposite directions.

Assuming constant magnetic flux in the detector coil we have

$$
\delta J_{z} A_{r}=\mu_{0} i_{s}(N / l) A_{c}
$$

where $A_{r}$ is the cross-section area of the ribbon and $A_{c}$ is the area of the coil. $i_{s}$ is the short-circuit current due to $\delta J_{z}$ and $N / l$ is the number of turns per unit length. Let $i_{H}=H(N / l)^{-1}$ be the current necessary to cancel $H$. Then we define the figure of merit as $i_{s} / i_{H}$ and obtain

$$
i_{s} / i_{H}=\left(A_{r} / A_{c}\right)\left(\delta J_{z} / \mu_{0} H\right)=\left(A_{r} / A_{c}\right) D^{-1}
$$


which shows the importance of keeping $D$ low as is the case for the ribbon dimension used in this sensor.

\section{ConCLUSIONS}

We have proposed and tested a fluxgate sensor which can be characterized as an extremely simple construction consisting of only one coil surrounding a heat-treated metallic glass ribbon formed in a hairpin shape. The construction is based on the ability of inducing, by torsional stress annealing, a helical anisotropy in a zero-magnetostriction metallic glass ribbon. The sensor performance is similar to a traditional fluxgate sensor but, in contrast to the latter, the miniaturization is straightforward because of the simplicity. Finally, we emphasize that the present sensors have been made by choosing the preparation parameters (annealing conditions) from "first assessment." Further investigations are in progress in order to reduce the noise level by choosing more suitable preparation parameters.

\section{REFERENCES}

[1] D. I. Gordon and R. E. Brown, "Recent advances in fluxgate magnetometry," IEEE Trans. Magn., vol. MAG-8, pp. 76-82, Mar. 1972.

[2] F. Primdahl, "The fluxgate mechanism, part I: The gating curves of parallel and orthogonal fluxgates," IEEE Trans. Magn., vol. MAG6, pp. 376-383, June 1970.

[3] R. D. Wyckoff, "The gulf airborne magnetometer," Geophys., vol. 13, pp. 182-208, Apr. 1948

[4] R. Kuhne, "Magnetfeldmessung mit Eisenkern-Magnetometer nach dem Oberwellenverfaren,' ATM Archiv fur Technisches Messen, vol 392-1, Lieferung 199, DK 621.317.42, pp. 175-178, Aug. 1952.

[5] T. M. Palmer, “A small sensitive magnetometer," Proc. Inst. Elec. Eng., vol. 100, pt. II, pp. 545-550, 1953.
[6] L. R. Alldredge, "Magnetometer," U.S. Patent 2856581 , Oct. 14, 1958.

[7] E. O. Schonstedt, "Saturable measuring device and magnetic core therefore," U.S. Patent 2916 696, Dec. 8, 1959, also U.S. Patent 981885 , Apr. 25, 1961

[8] T. H. O'Dell, "Magnetostriction measurements on amorphous ribbons by the Becker-Kersten method,' Phys. Stat. Solidi (a), vol. 68 pp. 221-228, 1981

[9] O. V. Nielsen, J. R. Petersen, B. Hernando, J.Gutierrez, and F. Primdahl, "Metallic glasses for fluxgate applications," Anales de Fisica, in press.

[10] O. V. Nielsen, A. Hernando, V. Madurga, and J. M. Gonzalez, “Experiments concerning the origin of stress anneal induced magnetic anisotropy in metallic glass ribbons," J. Magn. Magn. Mat., vol. 46, pp. $341-349,1985$

[11] O. V. Nielsen, H. J. V. Nielsen, T. Masumoto, and H. M. Kimura "Correlation between magnetic anisotropy induced by stress anneal ing and viscoelastic properties for a metallic glass ribbon," J. Magn. Magn. Mat., vol. 24, pp. 88-92, 1981.

[12] J. D. Livingston and W. G. Morris, "Magnetic domains in twisted amorphous ribbons," IEEE Trans. Magn., vol. MAG-20, pp. 1379$1381,1984$.

[13] F. Primdahl, J. R. Petersen, C. Olin, and K. Harbo Andersen, "The short-circuited fluxgate output current,' J. Phys. E: Sci. Instrum., vol. 22 , pp. 349-354, 1989

Otto V. Nielsen, photograph not available at the time of publication.

J. Gutierrez, photograph not available at the time of publication.

B. Hernando, photograph not available at the time of publication.

Howard T. Savage, biography not available at the time of publication. 